Korean J. Math. 19 (2011), No. 2, pp. 191-203

\title{
LARGE AMPLITUDE THEORY OF A SHOCK-ACCELERATED INSTABILITY IN COMPRESSIBLE FLUIDS
}

\author{
SUNG-IK SOHN
}

\begin{abstract}
The interface between fluids of different densities is unstable under acceleration by a shock wave. A previous small amplitude linear theory for the compressible Euler equation failed to provide a quantitatively correct prediction for the growth rate of the unstable interface. In this paper, to include dominant nonlinear effects in a large amplitude regime, we present high-order perturbation equations of the Euler equation, and boundary conditions for the contact interface and shock waves.
\end{abstract}

\section{Introduction}

When an incident shock collides with an interface between two fluids of different densities, the interface becomes hydrodynamically unstable, while the shock bifurcates into a transmitted shock and a reflected wave [1]. This interfacial instability is known as RichtmyerMeshkov (RM) instability) and plays an important role in many fields ranging from astrophysics to inertial confinement fusion [2]. The RM instability has been studied in a wide range of contexts, from theories $[3,4,5,6,7,8,9,10,11]$ to numerical simulations $[12,13,14,15]$.

The dominant characteristics of the RM unstable interface are fingers, known as bubble and spike, of each phase extending into the region occupied by the opposite phase [2]. Thus a bubble (spike) is a portion of the light (heavy) fluid penetrating into the heavy (light) fluid. Eventually,

Received April 25, 2011. Revised June 18, 2011. Accepted June 20, 2011.

2000 Mathematics Subject Classification: 76E17, 76E30, 76L05.

Key words and phrases: interfacial instability, Richtmyer-Meshkov instability, perturbation theory, shock wave.

This research was supported by Basic Science Research Program through the National Research Foundation of Korea (NRF) funded by the Ministry of Education, Science and Technology (Grant No. 2011-0005263). 
the perturbation on the interface develops to a turbulent mixing of two fluids.

Richtmyer [1] proposed a small amplitude linear theory for the compressible Euler equation for the RM instability, and predicted the constant growth rate of the interface. However, results of numerical simulations revealed a decaying growth of the RM interface at a late time [13, 15]. Recently, potential flow models [8, 9] showed that the RM interface has an asymptotic growth rate of the form $1 /(k t)$, where $k$ is the wave number of the interface.

Besides the potential flow models, perturbation methods have been studied by several authors $[4,5,10,11]$, to describe the early and intermediate nonlinear regimes of the RM instability. This line of the methods is called as the weakly nonlinear theory, and was first proposed by Zhang and Sohn [4], extending the linear impulsive model by Richtmyer [1]. Vandenboomgaerde and his collaborators [10, 11] further developed the weakly nonlinear theory and found an efficient method.

Although the potential flow models and the weakly nonlinear theory give good descriptions for nonlinearity of the evolution of the RM interface, these models are based on the assumption of incompressible fluids, and therefore neglect compressibility effects. To include both effects of compressibility and nonlinearity, a large amplitude theory for the compressible Euler equation is called for, but the compressible Euler equation is still limited to the linear theory, and fails to give a quantitatively correct prediction for the growth rate of the unstable interface.

In this paper, we present high-order equations of the compressible Euler equation, and boundary conditions for the contact interface and shocks for the RM instability, extending the linearized Euler equation by Richtmyer [1]. The second-order perturbations of the Euler equation and boundary conditions are obtained, to include dominant nonlinear effects in a large amplitude regime.

In Section 2, the zeroth order solution and first-order linear theory is summarized. We present the high-order perturbation equations for the fluids in Section3, and the high-order boundary conditions in Section 4. Section 5 gives concluding remarks. 


\section{Linear equations}

In this section, we summarize the equations and boundary conditions for the small amplitude theory of the RM instability. These equations are derived by linearizing the compressible Euler equation [1].

The Euler equation is given by

$$
\begin{aligned}
\frac{\partial v}{\partial t}+u_{x} \frac{\partial v}{\partial x}+u_{z} \frac{\partial v}{\partial z} & =v\left(\frac{\partial u_{x}}{\partial x}+\frac{\partial u_{z}}{\partial z}\right), \\
\frac{\partial u_{x}}{\partial t}+u_{x} \frac{\partial u_{x}}{\partial x}+u_{z} \frac{\partial u_{x}}{\partial z} & =-v \frac{\partial p}{\partial x} \\
\frac{\partial u_{z}}{\partial t}+u_{x} \frac{\partial u_{z}}{\partial x}+u_{z} \frac{\partial u_{z}}{\partial z} & =-v \frac{\partial p}{\partial z}
\end{aligned}
$$

with the equation of state $p=f(v, s)$, where the fluid velocity is denoted by $\mathbf{u}=\left(u_{x}, u_{z}\right)$, the specific volume $v$, and the specific entropy $s$.

2.1. Riemann solution for shock-contact interaction. The zeroth order solution of the RM instability is the unperturbed problem of the shock-contact interaction, i.e. a head-on collision of a plane shock with a flat contact discontinuity. This shock-contact interaction produces a transmitted shock, and a reflected wave that can be either a shock or a rarefaction. The shock-contact interaction defines a one-dimensional Riemann problem for compressible fluids [16].

The solution of the one-dimensional Riemann problem can be analyzed by the pressure-velocity wave diagram [3, 16]. For a given state of the fluid ahead of a shock, there exist a family of two different solutions for the fluid state behind the shock, i.e. the shock branch and the rarefaction branch. The Riemann solution can be obtained using a wave diagram by finding the intersection of the wave curves coming out of the behind states of the contact and the incident shock. The outcome of the shock-contact interaction is determined by the adiabatic exponents $\gamma_{0}$, and $\gamma_{1}$, the ratio of the specific volume $v_{1} / v_{0}$, and the incident shock strength.

2.2. First-order equations. We now assume that the plane shock wave collides with the contact interface of a sinusoidal perturbation. The initial (pre-shocked) amplitude $a_{0}(0-)$ of the interface is the small expansion parameter for the problem.

After the shock-contact interaction, the domain is separated into four regions by the transmitted shock, the contact discontinuity, and the 
3

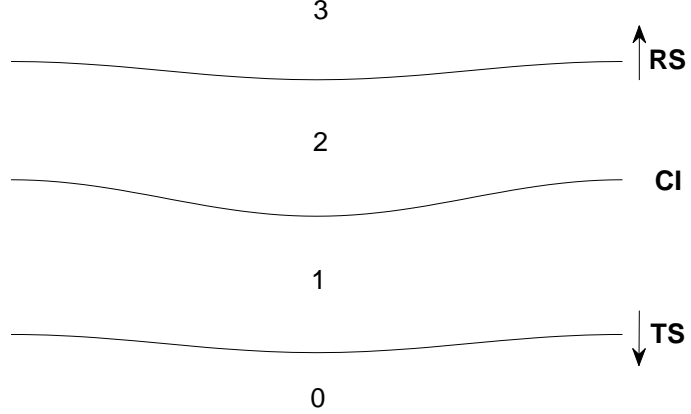

Figure 1. An illustration of the RM instability after the shock-contact interaction. The symbols CI, TS and RS represent the contact interface, the transmitted shock and the reflected shock, respectively.

reflected shock (See Figure 1). We label these regions 0 to 3, from bottom to top. Referring to Fig. 1, we take a co-moving coordinate, in which the mean position of the contact interface is $z=0$. Let $w_{1}$ and $w_{2}$ be the mean speeds of the transmitted and reflected shock. Then the positions of the transmitted shock, interface, and reflected shock is represented by

$$
\begin{array}{rrr}
z= & -w_{1} t+a_{1}(t) \cos k x, \\
z= & a_{0}(t) \cos k x, \\
z= & w_{2} t+a_{2}(t) \cos k x,
\end{array}
$$

where $k$ is the wave number of the perturbation.

In regions 0 and 3, all perturbed quantities are of zeroth order. In the linear theory, any quantity in region 1 and 2 can be expressed as

$$
Q(z, t)=Q^{0}+Q^{1}(z, t) \cos k x .
$$

Since the zeroth-order solutions for region 1 and 2 are constant states, it is easy to show that the pressure disturbances satisfy the wave equation in these two regions,

$$
\frac{\partial^{2} p^{1}}{\partial t^{2}}=c_{l}^{2}\left(\frac{\partial^{2} p^{1}}{\partial z^{2}}-k^{2} p^{1}\right)
$$


where $c_{l}$ is the sound speed of the fluid, and the subscript indicates the regions, $l=1,2$.

In addition to Eq. (8), we need the boundary conditions at the contact interface and shock waves. On the contact interface, the continuity of the normal component of the fluid acceleration gives

$$
\ddot{a}_{0}(t)=-\left.v_{1}^{0} \frac{\partial p^{1}}{\partial z}\right|_{z=0-}=-\left.v_{2}^{0} \frac{\partial p^{1}}{\partial z}\right|_{z=0+} .
$$

At the transmitted shock, we have

$$
\begin{aligned}
\dot{a}_{1}(t) & =-C_{t s 1} p^{1}\left(-w_{1} t, t\right) \\
\frac{d p^{1}\left(-w_{1} t, t\right)}{d t} & =-\left.C_{t s 2} \frac{\partial p^{1}}{\partial z}\right|_{z=-w_{1} t}+C_{t s 3} a_{1}(t),
\end{aligned}
$$

where the coefficients are defined as

$$
\begin{aligned}
C_{t s 1} & =\frac{1}{2} \frac{v_{0}^{0} v_{1}^{0}}{v_{0}^{0}-v_{1}^{0}}\left(\frac{1}{w_{1}}-\frac{w_{1}}{K_{1} c_{1}^{2}}\right), \\
C_{t s 2} & =\left(w_{1}^{2}-c_{1}^{2}\right) /\left(w_{1}+\frac{c_{1}^{2}}{2 w_{1}}+\frac{w_{1}}{2 K_{1}}\right), \\
C_{t s 3} & =k^{2} c_{1}^{2} u_{0}^{0} w_{1}^{2} /\left(w_{1}+\frac{c_{1}^{2}}{2 w_{1}}+\frac{w_{1}}{2 K_{1}}\right) .
\end{aligned}
$$

Here, $K_{1}$ is the dimensionless slope of the Rankine-Hugoniot function evaluated at the state 1 for a given ahead state (state 0 ). If we denote the Rankine-Hugoniot function as $p_{h}(v)$, we have

$$
K_{1}=-\left.\left(\frac{v_{1}^{0}}{c_{1}}\right)^{2} \frac{d p_{h}(v)}{d v}\right|_{v=v_{1}^{0}} .
$$

The boundary conditions similar to Eqs. (10) and (11) hold at the reflected shock, and are given by

$$
\begin{aligned}
\dot{a_{2}(t)} & =C_{r s 1} p^{1}\left(w_{2} t, t\right), \\
\frac{d p^{1}\left(w_{2} t, t\right)}{d t} & =\left.C_{r s 2} \frac{\partial p^{1}}{\partial z}\right|_{z=w_{2} t}+C_{r s 3} a_{2}(t),
\end{aligned}
$$

where the coefficients $C_{r s j}, j=1,2,3$, are defined by changing all the subscripts 0 to 3 , and 1 to 2 in the expressions of $C_{t s j}$. 


\section{High-order equations}

We derive high order perturbation equations for the RM instability. For high-order equations, the second order harmonics are added in the expression (7). We found that, in addition to the second order harmonics, high-order translation terms should also be included in the expansions, due to interactions of first and high orders. In other words, any quantity in region 1 and 2 is expressed as

$$
Q(z, t)=Q^{0}+Q^{1}(z, t) \cos k x+\bar{Q}^{2}(z, t)+Q^{2}(z, t) \cos 2 k x .
$$

For region 1 , if $p=f_{1}(v, s)$ is the equation of state, then to second order,

$$
\begin{aligned}
& p^{2}(z, t)=-\left(\frac{c_{1}}{v_{1}^{0}}\right)^{2}\left(v^{2}+\left.s^{2} \frac{\frac{\partial f_{1}}{\partial s}}{\frac{\partial f_{1}}{\partial v}}\right|_{v_{1}^{0}, s_{1}^{0}}+\left.\frac{1}{4}\left(v^{1}\right)^{2} \frac{\frac{\partial^{2} f_{1}}{\partial v^{2}}}{\frac{\partial f_{1}}{\partial v}}\right|_{v_{1}^{0}, s_{1}^{0}}\right. \\
& \left.+\left.\frac{1}{2} v^{1} s^{1} \frac{\frac{\partial^{2} f_{1}}{\partial v \partial s}}{\frac{\partial f_{1}}{\partial v}}\right|_{v_{1}^{0}, s_{1}^{0}}+\left.\frac{1}{4}\left(s^{1}\right)^{2} \frac{\frac{\partial^{2} f_{1}}{\partial s^{2}}}{\frac{\partial f_{1}}{\partial v}}\right|_{v_{1}^{0}, s_{1}^{0}}\right), \\
& \bar{p}^{2}(z, t)=-\left(\frac{c_{1}}{v_{1}^{0}}\right)^{2}\left(\bar{v}^{2}+\left.\bar{s}^{2} \frac{\frac{\partial f_{1}}{\partial s}}{\frac{\partial f_{1}}{\partial v}}\right|_{v_{1}^{0}, s_{1}^{0}}+\left.\frac{1}{4}\left(v^{1}\right)^{2} \frac{\frac{\partial^{2} f_{1}}{\partial v^{2}}}{\frac{\partial f_{1}}{\partial v}}\right|_{v_{1}^{0}, s_{1}^{0}}\right. \\
& \left.+\left.\frac{1}{2} v^{1} s^{1} \frac{\frac{\partial^{2} f_{1}}{\partial v \partial s}}{\frac{\partial f_{1}}{\partial v}}\right|_{v_{1}^{0}, s_{1}^{0}}+\left.\frac{1}{4}\left(s^{1}\right)^{2} \frac{\frac{\partial^{2} f_{1}}{\partial s^{2}}}{\frac{\partial f_{1}}{\partial v}}\right|_{v_{1}^{0}, s_{1}^{0}}\right) \text {. }
\end{aligned}
$$

Then, to second order, the equation of continuity (1) is given by

$$
\begin{aligned}
\frac{\partial v^{2}}{\partial t}= & \frac{1}{2}\left(v^{1} \frac{\partial u_{z}^{1}}{\partial z}-u_{z}^{1} \frac{\partial v^{1}}{\partial z}\right)+v_{1}^{0}\left(-2 k u_{x}^{2}+\frac{\partial u_{z}^{2}}{\partial z}\right) \\
= & -\left(\frac{v_{1}^{0}}{c_{1}}\right)^{2} \frac{\partial p^{2}}{\partial t}-\frac{1}{2} \frac{\partial v^{1}}{\partial t}\left(\left.v^{1} \frac{\frac{\partial^{2} f_{1}}{\partial v^{2}}}{\frac{\partial f_{1}}{\partial v}}\right|_{v_{1}^{0}, s_{1}^{0}}+\left.s^{1} \frac{\frac{\partial^{2} f_{1}}{\partial s \partial s}}{\frac{\partial f_{1}}{\partial v}}\right|_{v_{1}^{0}, s_{1}^{0}}\right) \\
& +\left.\frac{1}{2}\left(u_{z}^{1} \frac{\partial s^{1}}{\partial z}-k u_{x}^{1} s^{1}\right) \frac{\frac{\partial f_{1}}{\partial s}}{\frac{\partial f_{1}}{\partial v}}\right|_{v_{1}^{0}, s_{1}^{0}},
\end{aligned}
$$


(19) $\frac{\partial \bar{v}^{2}}{\partial t}=\frac{1}{2}\left(v^{1} \frac{\partial u_{z}^{1}}{\partial z}-u_{z}^{1} \frac{\partial v^{1}}{\partial z}\right)-k v^{1} u_{x}^{1}+v_{1}^{0} \frac{\partial \bar{u}_{z}}{\partial z}$

$$
\begin{aligned}
= & -\left(\frac{v_{1}^{0}}{c_{1}}\right)^{2} \frac{\partial \bar{p}^{2}}{\partial t}-\frac{1}{2} \frac{\partial v^{1}}{\partial t}\left(\left.v^{1} \frac{\frac{\partial^{2} f_{1}}{\partial v^{2}}}{\frac{\partial f_{1}}{\partial v}}\right|_{v_{1}^{0}, s_{1}^{0}}+\left.s^{1} \frac{\frac{\partial^{2} f_{1}}{\partial s \partial s}}{\frac{\partial f_{1}}{\partial v}}\right|_{v_{1}^{0}, s_{1}^{0}}\right) \\
& +\left.\frac{1}{2}\left(u_{z}^{1} \frac{\partial s^{1}}{\partial z}+k u_{x}^{1} s^{1}\right) \frac{\frac{\partial f_{1}}{\partial s}}{\frac{\partial f_{1}}{\partial v}}\right|_{v_{1}^{0}, s_{1}^{0}},
\end{aligned}
$$

and the momentum equations (2) and (3) are

$$
\begin{aligned}
& \frac{\partial u_{x}^{2}}{\partial t}-\frac{k}{2}\left(u_{x}^{1}\right)^{2}+\frac{1}{2} u_{z}^{1} \frac{\partial u_{x}^{1}}{\partial z}=-2 k\left(v_{1}^{0} p^{2}+\frac{1}{4} v^{1} p^{1}\right) \\
& \frac{\partial u_{z}^{2}}{\partial t}-\frac{k}{2} u_{x}^{1} u_{z}^{1}+\frac{1}{2} u_{z}^{1} \frac{\partial u_{z}^{1}}{\partial z}=-\left(v_{1}^{0} \frac{\partial p^{2}}{\partial z}+\frac{1}{2} v^{1} \frac{\partial p^{1}}{\partial z}\right) \\
& \frac{\partial \bar{u}_{z}^{2}}{\partial t}+\frac{k}{2} u_{x}^{1} u_{z}^{1}+\frac{1}{2} u_{z}^{1} \frac{\partial u_{z}^{1}}{\partial z}=-\left(v_{1}^{0} \frac{\partial \bar{p}^{2}}{\partial z}+\frac{1}{2} v^{1} \frac{\partial p^{1}}{\partial z}\right) .
\end{aligned}
$$

Differentiating (18) with respect to $t$, we obtain the equation for $p^{2}$ and $\bar{p}$,

$$
\begin{aligned}
\frac{\partial^{2} p^{2}}{\partial t^{2}} & =c_{1}^{2}\left(\frac{\partial^{2} p^{2}}{\partial z^{2}}-4 k^{2} p^{2}\right)+\frac{1}{2} S, \\
\frac{\partial^{2} \bar{p}^{2}}{\partial t^{2}} & =c_{1}^{2} \frac{\partial^{2} p^{2}}{\partial z^{2}}+\frac{1}{2} \bar{S},
\end{aligned}
$$

where the source terms are

$$
\begin{aligned}
S= & \frac{\partial p^{1}}{\partial t} \frac{\partial u_{z}^{1}}{\partial z}-u_{z}^{1} \frac{\partial^{2} p^{1}}{\partial t \partial z}+\frac{c_{1}^{2}}{v_{1}^{0}}\left(2 k^{2}\left(\left(u_{x}^{1}\right)^{2}-v^{1} p^{1}\right)-3 k u_{z}^{1} \frac{\partial u_{x}^{1}}{\partial z}\right. \\
& \left.-k u_{x}^{1} \frac{\partial u_{z}^{1}}{\partial z}+\left(\frac{\partial u_{z}^{1}}{\partial z}\right)^{2}+u_{z}^{1} \frac{\partial^{2} u_{z}^{1}}{\partial z^{2}}+2 v^{1} \frac{\partial^{2} p^{1}}{\partial z^{2}}\right)+H_{0} \\
\bar{S}= & \frac{\partial p^{1}}{\partial t} \frac{\partial u_{z}^{1}}{\partial z}-u_{z}^{1} \frac{\partial^{2} p^{1}}{\partial t \partial z}-2 k \frac{\partial p^{1}}{\partial t} u_{x}^{1}+\frac{c_{1}^{2}}{v_{1}^{0}}\left(k\left(\frac{\partial u_{x}^{1}}{\partial z} u_{z}^{1}+u_{x}^{1} \frac{\partial u_{z}^{1}}{\partial z}\right)\right. \\
& \left.-2 k^{2} v^{1} p^{1}+\left(\frac{\partial u_{z}^{1}}{\partial z}\right)^{2}+u_{z}^{1} \frac{\partial^{2} u_{z}^{1}}{\partial z^{2}}+2 v^{1} \frac{\partial^{2} p^{1}}{\partial z^{2}}\right)+H_{1},
\end{aligned}
$$


and

$$
\begin{aligned}
H_{i}= & -\left(\frac{c_{1}}{v_{1}^{0}}\right)^{2}\left[\frac{\partial^{2} v^{1}}{\partial t^{2}}\left(\left.v^{1} \frac{\frac{\partial^{2} f_{1}}{\partial v^{2}}}{\frac{\partial f_{1}}{\partial v}}\right|_{v_{1}^{0}, s_{1}^{0}}+\left.s^{1} \frac{\frac{\partial^{2} f_{1}}{\partial v \partial s}}{\frac{\partial f_{1}}{\partial v}}\right|_{v_{1}^{0}, s_{1}^{0}}\right)\right. \\
& \left.+\left.\left(\frac{\partial v^{1}}{\partial t}\right)^{2} \frac{\partial^{2} f_{1}}{\frac{\partial v^{2}}{\partial f_{1}}}\right|_{v_{1}^{0}, s_{1}^{0}}+v_{1}^{0}\left(\frac{\partial p^{1}}{\partial z} \frac{\partial s^{1}}{\partial z}-\left.(-1)^{i} k^{2} p^{1} s^{1} \frac{\frac{\partial f_{1}}{\partial s}}{\frac{\partial f_{1}}{\partial v}}\right|_{v_{1}^{0}, s_{1}^{0}}\right)\right],
\end{aligned}
$$

for $i=0,1$.

It is not surprising that the second-order equation (23) has the factor 4 in the bracket, and the source term. For region 2, Eqs. (23) and (24) similarly holds, changing $c_{1}, v_{1}^{0}, s_{1}^{0}$ and $f_{1}$, to $c_{2}, v_{2}^{0}, s_{2}^{0}$ and $f_{2}$, respectively.

\section{High-order boundary conditions}

In the high-order, the positions of the transmitted shock, interface, and reflected shock are expressed by

$$
\begin{array}{rrr}
z= & -w_{1} t+a_{1}(t) \cos k x+\bar{b}_{1}(t)+b_{1}(t) \cos 2 k x, \\
z= & a_{0}(t) \cos k x+\bar{b}_{0}(t)+b_{0}(t) \cos 2 k x, \\
z= & w_{2} t+a_{2}(t) \cos k x+\bar{b}_{2}(t)+b_{2}(t) \cos 2 k x .
\end{array}
$$

4.1. The contact interface. The continuity of the normal component of the fluid acceleration gives

$$
\begin{aligned}
& \ddot{b}_{0}(t)=-\left.v_{1}^{0} \frac{\partial p^{2}}{\partial z}\right|_{z=0-}-\frac{1}{2} F_{-}^{0}=-\left.v_{2}^{0} \frac{\partial p^{2}}{\partial z}\right|_{z=0+}-\frac{1}{2} F_{+}^{0}, \\
& \ddot{\bar{b}}_{0}(t)=-\left.v_{1}^{0} \frac{\partial \bar{p}^{2}}{\partial z}\right|_{z=0-}-\frac{1}{2} F_{-}^{1}=-\left.v_{2}^{0} \frac{\partial \bar{p}^{2}}{\partial z}\right|_{z=0+}-\frac{1}{2} F_{+}^{1}
\end{aligned}
$$

where

$$
\begin{gathered}
F_{-}^{i}=\left.v^{1} \frac{\partial p^{1}}{\partial z}\right|_{z=0-}+\left.a_{0}(t) v_{1}^{0} \frac{\partial^{2} p^{1}}{\partial z^{2}}\right|_{z=0-}+\left.\dot{a}_{0}(t)\left((-1)^{i} k u_{x}^{1}+\frac{\partial u_{z}^{1}}{\partial z}\right)\right|_{z=0-}, \\
F_{+}^{i}=\left.v^{1} \frac{\partial p^{1}}{\partial z}\right|_{z=0+}+\left.a_{0}(t) v_{1}^{0} \frac{\partial^{2} p^{1}}{\partial z^{2}}\right|_{z=0+}+\left.\dot{a}_{0}(t)\left((-1)^{i} k u_{x}^{1}+\frac{\partial u_{z}^{1}}{\partial z}\right)\right|_{z=0+},
\end{gathered}
$$

for $i=0,1$. 
4.2. The shock waves. Expanding the jump condition at the transmitted shock to second order, we have

$$
\begin{aligned}
& \text { (30) } \frac{1}{v_{1}^{0}}\left[\dot{b}_{1}-\frac{k^{2}}{4} a_{1}^{2} w_{1}-u_{z}^{2}-\frac{a_{1}}{2}\left(\frac{\partial u_{z}^{1}}{\partial z}-k u_{x}^{1}\right)\right]+\frac{1}{2} \frac{v^{1}}{\left(v_{1}^{0}\right)^{2}}\left(u_{z}^{1}-\dot{a}_{1}\right) \\
& +\frac{w_{1}}{\left(v_{1}^{0}\right)^{3}}\left[v_{1}^{0}\left(v^{2}+\frac{a_{1}}{2} \frac{\partial v^{1}}{\partial z}\right)-\frac{\left(v^{1}\right)^{2}}{2}\right]=\frac{1}{2} \sqrt{\frac{p_{1}^{0}-p_{0}^{0}}{v_{0}^{0}-v_{1}^{0}}}\left[\frac{p^{2}+\frac{a_{1}}{2} \frac{\partial p^{1}}{\partial z}}{p_{1}^{0}-p_{0}^{0}}\right. \\
& \left.+\frac{v^{2}+\frac{a_{1}}{2} \frac{\partial v^{1}}{\partial z}}{v_{0}^{0}-v_{1}^{0}}-\frac{1}{8} \frac{\left(p^{1}\right)^{2}}{\left(p_{1}^{0}-p_{0}^{0}\right)^{2}}+\frac{1}{4} \frac{p^{1} v^{1}}{\left(p_{1}^{0}-p_{0}^{0}\right)\left(v_{0}^{0}-v_{1}^{0}\right)}+\frac{3}{8} \frac{\left(v^{1}\right)^{2}}{\left(v_{0}^{0}-v_{1}^{0}\right)^{2}}\right], \\
& \frac{1}{v_{0}^{0}}\left[\dot{b}_{1}-\frac{1}{4} k^{2} a_{1}^{2}\left(w_{1}+u_{0}^{0}\right)\right]=R,
\end{aligned}
$$

and

$$
\begin{aligned}
& \text { (32) } \frac{1}{v_{1}^{0}}\left[\dot{\bar{b}}_{1}+\frac{k^{2}}{4} a_{1}^{2} w_{1}-\bar{u}_{z}^{2}-\frac{a_{1}}{2}\left(\frac{\partial u_{z}^{1}}{\partial z}+k u_{x}^{1}\right)\right]+\frac{1}{2} \frac{v^{1}}{\left(v_{1}^{0}\right)^{2}}\left(u_{z}^{1}-\dot{a}_{1}\right) \\
& +\frac{w_{1}}{\left(v_{1}^{0}\right)^{3}}\left[v_{1}^{0}\left(\bar{v}^{2}+\frac{a_{1}}{2} \frac{\partial v^{1}}{\partial z}\right)-\frac{\left(v^{1}\right)^{2}}{2}\right]=\frac{1}{2} \sqrt{\frac{p_{1}^{0}-p_{0}^{0}}{v_{0}^{0}-v_{1}^{0}}}\left[\frac{\bar{p}^{2}+\frac{a_{1}}{2} \frac{\partial p^{1}}{\partial z}}{p_{1}^{0}-p_{0}^{0}}\right. \\
& \left.+\frac{\bar{v}^{2}+\frac{a_{1}}{2} \frac{\partial v^{1}}{\partial z}}{v_{0}^{0}-v_{1}^{0}}-\frac{1}{8} \frac{\left(p^{1}\right)^{2}}{\left(p_{1}^{0}-p_{0}^{0}\right)^{2}}+\frac{1}{4} \frac{p^{1} v^{1}}{\left(p_{1}^{0}-p_{0}^{0}\right)\left(v_{0}^{0}-v_{1}^{0}\right)}+\frac{3}{8} \frac{\left(v^{1}\right)^{2}}{\left(v_{0}^{0}-v_{1}^{0}\right)^{2}}\right], \\
& \frac{1}{v_{0}^{0}}\left[\dot{\bar{b}}_{1}+\frac{1}{4} k^{2} a_{1}^{2}\left(w_{1}+u_{0}^{0}\right)\right]=\bar{R},
\end{aligned}
$$

where $R$ and $\bar{R}$ represent the right hand sides of Eqs. (30) and (32), respectively.

We define the dimensionless curvature of the Rankine-Hugoniot function as

$$
G_{1}=-\left.\frac{\left(v_{1}^{0}\right)^{3}}{\left(c_{1}\right)^{2}} \frac{d^{2} p_{h}(v)}{d v^{2}}\right|_{v=v_{1}^{0}} .
$$

Then Eqs. (30) and (32) give one set of boundary conditions

$$
\begin{aligned}
& \dot{b_{1}}(t)=-\left.C_{t s 1}\left(p^{2}+\frac{1}{2} a_{1} \frac{\partial p^{1}}{\partial z}\right)\right|_{z=-w_{1} t}-A+\frac{1}{4} a_{1}^{2} k^{2}\left(w_{1}+u_{0}^{0}\right), \\
& \dot{\bar{b}}_{1}(t)=-\left.C_{t s 1}\left(\bar{p}^{2}+\frac{1}{2} a_{1} \frac{\partial p^{1}}{\partial z}\right)\right|_{z=-w_{1} t}-A-\frac{1}{4} a_{1}^{2} k^{2}\left(w_{1}+u_{0}^{0}\right),
\end{aligned}
$$


where

$$
\begin{aligned}
A= & \frac{1}{8} \frac{v_{0}^{0} w_{1}}{v_{1}^{0}}\left[-\frac{1}{2} \frac{\left(p^{1}\right)^{2}}{\left(p_{1}^{0}-p_{0}^{0}\right)^{2}}+\frac{p^{1} v^{1}}{\left(p_{1}^{0}-p_{0}^{0}\right)\left(v_{0}^{0}-v_{1}^{0}\right)}+\frac{3}{2} \frac{\left(v^{1}\right)^{2}}{\left(v_{0}^{0}-v_{1}^{0}\right)^{2}}\right. \\
& \left.-\frac{\left(v^{1}\right)^{2}}{v_{1}^{0}\left(v_{0}^{0}-v_{1}^{0}\right)} \frac{G_{1}}{K_{1}}\right] .
\end{aligned}
$$

Equations (30) and (35) yield the expression for $u_{z}^{2}\left(-w_{1} t, t\right)$, and then taking the material derivative, we have

$$
\frac{d}{d t}\left(u_{z}^{2}+\frac{a_{1}}{2} \frac{\partial u_{z}^{1}}{\partial z}\right)=-\frac{v_{1}^{0}}{2}\left(\frac{1}{w_{1}}+\frac{w_{1}}{K_{1} c_{1}^{2}}\right) \frac{d}{d t}\left(p^{2}+\frac{a_{1}}{2} \frac{\partial p^{1}}{\partial z}\right)+B
$$

where

$$
\begin{aligned}
& B=\frac{3}{2} a_{1} \dot{a}_{1} k^{2} u_{0}^{3}+D, \\
& D=\frac{1}{2 v_{1}^{0}} \frac{d}{d t}\left(v^{1}\left(u_{z}^{1}+C_{t s 1} p^{1}\right)\right)-\frac{w_{1}}{\left(v_{1}^{0}\right)^{2}} v^{1} \frac{d v^{1}}{d t} \\
& -\frac{1}{8} \frac{\left(v_{0}^{0}-v_{1}^{0}\right) w_{1}}{v_{1}^{0}}\left[-\frac{p^{1}}{\left(p_{1}^{0}-p_{0}^{0}\right)^{2}} \frac{d p^{1}}{d t}+\frac{1}{\left(p_{1}^{0}-p_{0}^{0}\right)\left(v_{0}^{0}-v_{1}^{0}\right)} \frac{d\left(p^{1} v^{1}\right)}{d t}\right. \\
& \left.+\frac{1}{v_{0}^{0}-v_{1}^{0}}\left(\frac{3}{v_{0}^{0}-v_{1}^{0}}+\frac{8 G_{1}}{K_{1} v_{1}^{0}}\right) v^{1} \frac{d v^{1}}{d t}\right] \text {. }
\end{aligned}
$$

Similarly, for $\bar{u}_{z}^{2}\left(-w_{1} t, t\right)$, it gives

(38) $\frac{d}{d t}\left(\bar{u}_{z}^{2}+\frac{a_{1}}{2} \frac{\partial u_{z}^{1}}{\partial z}\right)=-\frac{v_{1}^{0}}{2}\left(\frac{1}{w_{1}}+\frac{w_{1}}{K_{1} c_{1}^{2}}\right) \frac{d}{d t}\left(\bar{p}^{2}+\frac{a_{1}}{2} \frac{\partial p^{1}}{\partial z}\right)+\bar{B}$,

where

$$
\bar{B}=-\frac{3}{2} a_{1} \dot{a}_{1} k^{2} u_{0}^{3}+D .
$$

The continuity of the tangential component of the fluid velocity gives the expression for $u_{x}^{2}\left(-w_{1} t, t\right)$,

$$
u_{x}^{2}\left(-w_{1} t, t\right)=2 k u_{0}^{0} b_{1}(t)+\frac{1}{2}\left(k u_{z}^{1}-\frac{\partial u_{x}^{1}}{\partial z}\right) a_{1}(t)
$$

Combining Eqs. (37)-(39), and using the equation of continuity and the momentum equations, we obtain the other set of boundary conditions

$$
\frac{d p^{2}\left(-w_{1} t, t\right)}{d t}=-C_{t s 2} \frac{\partial p^{2}}{\partial z}+4 C_{t s 3} b_{1}(t)+C_{t s 4}(-P-T+I)
$$


(41)

$$
\frac{d \bar{p}^{2}\left(-w_{1} t, t\right)}{d t}=-C_{t s 2} \frac{\partial \bar{p}^{2}}{\partial z}+C_{t s 4}(-P-T+\bar{I}),
$$

where

$$
\begin{aligned}
P= & \frac{1}{4}\left(\frac{c_{1}^{2}}{w_{1}}+\frac{w_{1}}{K_{1}}\right)\left[\dot{a}_{1} \frac{\partial p^{1}}{\partial z}+a_{1}\left(\frac{\partial^{2} p^{1}}{\partial t \partial z}-w_{1} \frac{\partial^{2} p^{1}}{\partial z^{2}}\right)\right], \\
T= & \frac{1}{2} \frac{c_{1}^{2}}{v_{1}^{0}}\left[\dot{a}_{1} \frac{\partial u_{z}^{1}}{\partial z}+a_{1}\left(\frac{\partial^{2} u_{z}^{1}}{\partial t \partial z}-w_{1} \frac{\partial^{2} u_{z}^{1}}{\partial z^{2}}\right)\right], \\
I= & \frac{c_{1}^{2}}{v_{1}^{0}}\left[\frac{1}{2}\left(v^{1} \frac{\partial p^{1}}{\partial z}-k u_{x}^{1} u_{z}^{1}+u_{z}^{1} \frac{\partial u_{z}^{1}}{\partial z}\right)+B-\left(\frac{\partial u_{x}^{1}}{\partial z}-k u_{z}^{1}\right) k w_{1} a_{1}\right] \\
& -\frac{w_{1}}{2}\left(\frac{c_{1}}{v_{1}^{0}}\right)^{2}\left[\frac{\partial v^{1}}{\partial t}\left(\left.v^{1} \frac{\frac{\partial^{2} f_{1}}{\partial v^{2}}}{\frac{\partial f_{1}}{\partial v}}\right|_{v_{1}^{0}, s_{1}^{0}}+\left.s^{1} \frac{\frac{\partial^{2} f_{1}}{\partial v \partial s}}{\frac{\partial f_{1}}{\partial v}}\right|_{v_{1}^{0}, s_{1}^{0}}\right)\right. \\
& \left.\left.-\left.\left(u_{z}^{1} \frac{\partial s^{1}}{\partial z}-k u_{x}^{1} s^{1}\right) \frac{\frac{\partial f_{1}}{\partial s}}{\frac{\partial f_{1}}{\partial v}}\right|_{v_{1}^{0}, s_{1}^{0}}+v^{1} \frac{\partial u_{z}^{1}}{\partial z}-u_{z}^{1} \frac{\partial v^{1}}{\partial z}\right)\right], \\
\bar{I}= & \frac{c_{1}^{2}}{v_{1}^{0}}\left[\frac{1}{2}\left(v^{1} \frac{\partial p^{1}}{\partial z}+k u_{x}^{1} u_{z}^{1}+u_{z}^{1} \frac{\partial u_{z}^{1}}{\partial z}\right)+\bar{B}\right. \\
& -\frac{w_{1}}{2}\left(\frac{c_{1}}{v_{1}^{0}}\right)^{2}\left[\frac{\partial v^{1}}{\partial t}\left(\left.v^{1} \frac{\frac{\partial^{2} f_{1}}{\partial v^{2}}}{\frac{\partial f_{1}}{\partial v}}\right|_{v_{1}^{0}, s_{1}^{0}}+\left.s^{1} \frac{\frac{\partial^{2} f_{1}}{\partial v \partial s}}{\frac{\partial f_{1}}{\partial v}}\right|_{v_{1}^{0}, s_{1}^{0}}\right)\right. \\
& \left.\left.-\left.\left(u_{z}^{1} \frac{\partial s^{1}}{\partial z}+k u_{x}^{1} s^{1}\right) \frac{\frac{\partial f_{1}}{\partial s}}{\frac{\partial f_{1}}{\partial v}}\right|_{v_{1}^{0}, s_{1}^{0}}+v^{1} \frac{\partial u_{z}^{1}}{\partial z}-u_{z}^{1} \frac{\partial v^{1}}{\partial z}-2 k v^{1} u_{x}^{1}\right)\right]
\end{aligned}
$$

and the coefficient

$$
C_{t s 4}=1 /\left(w_{1}+\frac{c_{1}^{2}}{2 w_{1}}+\frac{w_{1}}{2 K_{1}}\right) .
$$

The boundary conditions for the reflected shock can be derived similarly, and is omitted.

\section{Concluding remarks}

We have derived the second-order perturbation equations for the fluids, and boundary conditions for the contact interface and shocks, for 
the RM instability. The resulting equations are much more complicated than the linear equations, especially at the shocks.

It is found that the high-order translation terms appear in the perturbation expansions. To the author's knowledge, the high-order translation in the RM instability was not known, and might be important in the evaluation of the growth of the interface in the numerical simulation. In the numerical simulation, the amplitudes of the bubble and spike are calculated by subtracting the positions of the bubble and spike by the Riemann solutions. The evaluation of the amplitude of the bubble and spike in that way does not account the effect of the high-order translation of the interface, and thus might estimate the amplitude of the interface inaccurately.

We expect that the second-order amplitude $b_{0}(t)$ makes the growth of the interface diminish. To observe the behavior of the solution, we need to integrate the equations numerically. Due to the complexity of the equations, the numerical procedure also requires considerable works, and is under study now.

\section{References}

[1] R. D. Richtmyer, Taylor instability in shock acceleration of compressible fluids, Comm. Pure Appl. Math. 13 (1960), 297-319.

[2] D. Sharp, An overview of Rayleigh-Taylor instability, Physica D 12 (1984), 3-10.

[3] Y. Yang, Q. Zhang and D. H. Sharp, Small amplitude theory of RichtmyerMeshkov instability, Phys. Fluids A 6 (1994), 1856-1873.

[4] Q. Zhang and S.-I. Sohn, Nonlinear theory of unstable fluid mixing driven by shock waves, Phys. Fluids 9 (1997), 1106-1124.

[5] Q. Zhang and S.-I. Sohn, Quantitative theory of Richtmyer-Meshkov instability in three dimensions, Zeit. angew. Math. Phys. 50 (1999), 1-46.

[6] S.-I. Sohn, Computation and analysis of mathematical model for moving free boundary flows, J. Korean Math. Soc. 37 (2000), 779-791.

[7] B. Cheng, J. Glimm and D. H. Sharp, Dynamic evolution of Rayleigh-Taylor and Richtmyer-Meshkov mixing fronts, Phys. Rev. E 66 (2002), 036312: 1-7.

[8] S.-I. Sohn, Density dependence of a Zufiria-type model for Rayleigh-Taylor and Richtmyer-Meshkov bubble fronts, Phys. Rev. E 70 (2004), 045301: 1-4.

[9] S.-I. Sohn, Analytic solutions of unstable interfaces for all density ratios in axisymmetric flows, J. Comput. Appl. Math. 177 (2005), 367-374.

[10] M. Vandenboomgaerde, S. Gauthier and C. Mügler, Nonlinear regime of a multimode Richtmyer.Meshkov instability: A simplified perturbation theory, Phys. Fluids 14 (2002), 1111-1122. 
[11] M. Vandenboomgaerde, C. Cherfils, D. Galmiche, S. Gauthier and P. A. Raviart, Efficient perturbation methods for Richtmyer-Meshkov and Rayleigh-Taylor instabilities: Weakly nonlinear stage and beyond, Laser Part. Beams 21 (2003), 321-325.

[12] J. Grove, Applications of front tracking to the simulations of shock refractions and unstable mixing, Appl. Numer. Math. 14 (1994), 213-237.

[13] R. L. Holmes, J. W. Grove and D. H. Sharp, A numerical investigation of Richtmyer-Meshkov instability using front tracking, J. Fluid Mech. 301 (1995), 51-64.

[14] S.-I. Sohn, Analycal and numerical study of mode interactions in shock-induced interfacial instability, Commun. Korean Math. Soc. 15 (2000), 155-172.

[15] S.-I. Sohn, Vortex simulations of impulsively accelerated unstable interface, Math. Comput. Modelling 04 (2004), 627-636.

[16] R. Courant and K. O. Friedrich, Supersonic flow and shock waves, Springer, New York, 1976.

Department of Mathematics

Gangneung-Wonju National University

Gangneung 210-702, Korea

E-mail: sohnsi@gwnu.ac.kr 